\title{
Effects of boundary conditions on irreversible dynamics
}

\author{
Aldo Procacci ${ }^{1}$ \\ Benedetto Scoppola ${ }^{2}$ \\ March 14, 2017 \\ ${ }^{1}$ Departamento de Matemática, Universidade Federal de Minas Gerais \\ Av. António Carlos, 6627 - 30161-970 Belo Horizonte, Brazil \\ aldo@mat.ufmg.br \\ 2 Dipartimento di Matematica, Università di Roma "Tor Vergata" \\ Via della Ricerca Scientifica - 00133 Roma, Italy \\ scoppola@mat.uniroma2.it \\ ${ }^{3}$ Dipartimento di Matematica e Fisica, Università Roma Tre \\ Largo San Murialdo, 1 - 00146 Roma, Italy \\ scoppola@mat.uniroma3.it
}

Elisabetta Scoppola ${ }^{3}$

\begin{abstract}
We present a simple one-dimensional Ising-type spin system on which we define a completely asymmetric Markovian single spin-flip dynamics. We study the system at a very low, yet non-zero, temperature and we show that for empty boundary conditions the Gibbs measure is stationary for such dynamics, while introducing in a single site a + condition the stationary measure changes drastically, with macroscopical effects. We achieve this result defining an absolutely convergent series expansion of the stationary measure around the zero temperature system. Interesting combinatorial identities are involved in the proofs.
\end{abstract}




\section{Introduction}

In this paper we discuss a very simple one-dimensional spin system in order to point out the crucial effect of boundary conditions on the invariant measure of irreversible dynamics. Irreversible dynamics turn out to be a challenging problem since they are the main ingredient in the study of non-equilibrium statistical mechanics. Indeed many interesting physical systems can not be described in terms of equilibrium: for instance non-Hamiltonian evolutions, systems with external non-conservative forces, or systems with thermostats or reservoirs. Such systems exhibit non zero currents of matter or energy flowing in an irreversible way. For this kind of problems it is necessary to consider non-equilibrium statistical mechanics. Actually we can say that the description of non-equilibrium systems represents one of the "grand challenges" in statistical mechanics.

In this frame the main point is to describe Non-Equilibrium Stationary States (NESS), "in understanding the properties of states which are in stationary nonequilibrium: thus

establishing a clear separation between properties of evolution towards stationarity (or equilibrium) and properties of the stationary states themselves: a distinction which until the 1970's was rather blurred." as mentioned in the beautiful book by Gallavotti [7]. Irreversible dynamics play in this context a crucial role. The invariant measures of irreversible dynamics are stationary states but they describe non zero currents of probability, and hence they are NESS. A famous example is given by the TASEP model, in which particles hop only to the right, entering from a left reservoir with a given rate and leaving the system from the site $L$ with another rate.

In the context of Markovian dynamics, given any two states $i$ and $j$ in some configuration space $\mathcal{X}$, the irreversibility is defined by transition probabilities $P(i, j)$ violating the detailed balance condition

$$
\pi(j) P(j, i)=\pi(i) P(i, j) \quad \forall i, j \in \mathcal{X}
$$

This means that there are non zero probability currents. Indeed given a pair of states $i, j \in \mathcal{X}$ define the probability current (or flow of probability) from $j$ to $i$ at time $t$ the asymmetric function on $\mathcal{X} \times \mathcal{X}$ :

$$
K_{t}(j, i)=P^{t}(j) P(j, i)-P^{t}(i) P(i, j)
$$


where $P^{t}(\cdot)$ represents the probability of the state $\cdot$ at time $t$.

The continuity equation for $P^{t}(i)$, gives

$$
\begin{gathered}
P^{t+1}(i)-P^{t}(i)=\sum_{j} P^{t}(j) P(j, i)-P^{t}(i) \sum_{j} P(i, j)= \\
=\sum_{j \neq i}\left(P^{t}(j) P(j, i)-P^{t}(i) P(i, j)\right)=\sum_{j \neq i} K_{t}(j, i)=-\left(\operatorname{div} K_{t}\right)(i)
\end{gathered}
$$

Stationarity implies

$$
0=\sum_{j \neq i}(\pi(j) P(j, i)-\pi(i) P(i, j))=\sum_{j \neq i} K(j, i) \quad \forall i
$$

being $K(j, i)=\pi(j) P(j, i)-\pi(i) P(i, j)$, the stationary probability current (or stationary flow of probability) from $j$ to $i$, a divergence free flow. This flow $K$ is proportional to the antisymmetric part of the conductance associated to the chain and it is also considered for instance in [8]. Actually the presence of currents can be used to detect irreversible dynamics without using the invariant measure. This is done by the Kolmogorov criterion for reversibility [9]: the Markov dynamics with transition probabilities $P(i, j)$ is reversible if and only if for any loop of states: $i_{o}, i_{1}, i_{2}, \ldots, i_{n}, i_{o}$ we have

$$
P\left(i_{0}, i_{1}\right) P\left(i_{1}, i_{2}\right) \ldots . P\left(i_{n}, i_{0}\right)=P\left(i_{0}, i_{n}\right) \ldots . P\left(i_{2}, i_{1}\right) P\left(i_{1}, i_{0}\right) .
$$

This means that the dynamics is irreversible if there is a loop with a stationary current. As noted in the rich review by Chou, Mallick and Zia, [2], the presence of stationary current loops suggests to associate magnetostatics to irreversible dynamics as electrostatics is associated to reversible dynamics.

Beside their crucial role in the understanding of non-equilibrium statistical mechanics, irreversible dynamics have been frequently considered in the literature in order to speed up simulations. Indeed in some case rigorous control of mixing time of irreversible dynamics has been obtained. See for instance [5].

Several problems arise when considering irreversible dynamics. Indeed some tools frequently used in the study of convergence to equilibrium are strongly related to reversibility as spectral representation or the potential theoretical approach. Recently some progress 
has been done to extend some of these tools to non reversible dynamics. See for instance the extension of the Dirichlet principle to non reversible Markov chains obtained in [8]. In this paper we want to stress the main difficulty related to irreversibility: while detailed balance is a crucial tool to control the invariant measure of reversible dynamics, in the irreversible case the control of the invariant measure can be quite complicated, and in particular it is difficult to study its sensitivity to boundary conditions. Very recent results have been obtained in this direction in [6] where irreversible dynamics are constructed with a given Gibbsian stationary measure by exploiting cyclic decomposition of divergence free flows.

In some case it is possible to verify that the equation for the invariant measure (10) is satisfied by a suitable Gibbs measure, as proved below in the (easy) case of empty boundary conditions. This is also the case of 2-dimensional Ising model with asymmetric interaction discussed in [11] with periodic boundary condition. In general, due to the presence of probability currents, the verification of equation (10) typically involves non local argument and so the invariant measure strongly depends on boundary conditions.

We consider a one dimensional spin system on the discrete interval $[1, L] \equiv\{1,2, \ldots, L\}$ with a single-spin-flip Markovian dynamics $\left\{X_{t}\right\}_{t \in \mathbb{N}}$, defined on $\mathcal{X}:=\{-1,1\}^{L}$ by the following transition probabilities

$$
P\left(\sigma, \sigma^{(i)}\right)=\frac{1}{L} e^{-2 J\left(\sigma_{i} \sigma_{i-1}+1\right)}
$$

where $\sigma^{(i)}$ is the configuration obtained from $\sigma$ flipping the spin in the site $i \in\{1,2, \ldots, L\}$. This means that at each time a site $i$ is chosen uniformly at random in $\{1,2, \ldots, L\}$ and the spin is flipped in this site with probability one if it is opposite to its left neighbour, $\sigma_{i-1}$, or with probability $e^{-4 J}$ if it is parallel to $\sigma_{i-1}$. We will consider two different boundary conditions:

- the empty boundary condition corresponding to $\sigma_{0}=0$;

- the + boundary condition corresponding to $\sigma_{0}=+1$.

The chain is irreducible and aperiodic so that in both cases there exists a unique invariant measure. Our goal is to compare the invariant measures of the Markov chains corresponding to these two different boundary conditions in a very low temperature regime, i.e., when the parameter $J$ is sufficiently large w.r.t. $L$. 
We shall prove that while in the case of empty boundary conditions the stationary distribution is the Gibbs measure, in the case of + boundary condition the stationary measure changes drastically. Due to the particular low-temperature regime we are able to write the stationary distribution as an absolutely convergent expansion in $e^{-4 J}$. This expansion is easily controlled in this case, but it could be a general tool in order to control the invariant measure at a very low temperature in more general contexts. We control completely the first order of such expansion, and we show that it has several interesting features. In particular, the presence of probability currents implies that the boundary conditions do not have the effect of a conditioning, as in the case of the Gibbs measure. The boundary conditions actually modify the stationary distribution and the effect of their presence decay very slowly in the distance $i$ from the boundary, namely as $\frac{1}{\sqrt{i}}$. Moreover, the presence of boundary conditions makes the probabilities of interval of minus spins dependent on their length, producing macroscopical effects on the magnetization.

The paper is organized as follows: in section 2 we define the models, comparing them with the usual reversible Glauber Dynamics for the 1d Ising Model, and we state the main results of the paper. Section 3 is devoted to the control of the expansion of the invariant measure in terms of the quantity $e^{-4 J}$. Section 4 contains the proof related to the characterization of the first order term of the invariant measure. Some conclusion remarks and future perspectives are discussed in section 5 .

\section{Models and results}

As mentioned in the introduction, our model is defined via an irreversible markovian dynamics on a one dimensional discrete spin chain with states $\sigma \in \mathcal{X}=\{-1,+1\}\{1,2, \ldots, L\}$. We consider two different boundary conditions, namely the free boundary conditions, having $\sigma_{0}=\sigma_{L+1}=0$, and the + boundary condition $\sigma_{0}=\sigma_{L+1}=+1$. The dynamics is defined by the following transition matrix

$$
P^{I}(\sigma, \tau)= \begin{cases}\frac{1}{L} e^{-2 J\left(\sigma_{i} \sigma_{i-1}+1\right)} & \text { if } \tau=\sigma^{(i)} \\ 1-\frac{1}{L} \sum_{i} e^{-2 J\left(\sigma_{i} \sigma_{i-1}+1\right)} & \text { if } \tau=\sigma \\ 0 & \text { otherwise }\end{cases}
$$

where $\sigma^{(i)}$ is the configuration obtained from $\sigma$ by flipping the spin in the site $i$. This dynamics is irreversible, but in the case of free boundary condition it is easy to find its 
stationary measure. Indeed, consider the Gibbs measure

$$
\pi^{G}(\sigma)=\frac{e^{-H(\sigma)}}{Z^{G}}, \quad Z^{G}=\sum_{\sigma \in \mathcal{X}} e^{-H(\sigma)}
$$

where $H(\sigma)$ is the usual Ising Hamiltonian with free boundary conditions.

$$
H(\sigma)=-J \sum_{i=2}^{L} \sigma_{i} \sigma_{i-1}
$$

Let us show that $\pi^{G}(\sigma)$ is the unique stationary measure of dynamics (3). The dynamics is clearly irreducible and aperiodic, and hence the stationary measure exists and it is unique. Moreover it is immediate to verify the following equalities:

$$
\begin{gathered}
\pi^{G}\left(\sigma^{(i)}\right)=\pi^{G}(\sigma) e^{-2 J\left(\sigma_{i} \sigma_{i-1}+\sigma_{i} \sigma_{i+1}\right)} \\
P^{I}\left(\sigma^{(i)}, \sigma\right)=\frac{1}{L} e^{2 J\left(\sigma_{i} \sigma_{i-1}-1\right)}
\end{gathered}
$$

To prove that $\pi^{G}$ is the invariant measure of the process satisfying

$$
\sum_{\tau \in \mathcal{X}} \pi^{G}(\tau) P^{I}(\tau, \sigma)=\pi^{G}(\sigma)
$$

it is sufficient to verify the following condition, obtained by (8) by canceling the diagonal terms in both sides of the equality, which is equivalent to equation (10):

$$
\sum_{i=1}^{L} \pi^{G}\left(\sigma^{(i)}\right) P^{I}\left(\sigma^{(i)}, \sigma\right)=\pi^{G}(\sigma) \sum_{i=1}^{L} P^{I}\left(\sigma, \sigma^{(i)}\right)
$$

Equation (9) immediately follows from (6) and (7) since, by the empty b.c we have

$$
\sum_{i=1}^{L} e^{-2 J\left(\sigma_{i} \sigma_{i+1}\right)}=\sum_{i=1}^{L-1} e^{-2 J\left(\sigma_{i} \sigma_{i+1}\right)}=\sum_{i=2}^{L} e^{-2 J\left(\sigma_{i} \sigma_{i-1}\right)} .
$$

It is a standard task to define a reversible markovian dynamics having the same stationary measure, i.e. the well known Glauber dynamics, given by the following transition 
probability matrix

$$
P^{R}(\sigma, \tau)= \begin{cases}\frac{1}{L} e^{-\left[H\left(\sigma^{(i)}\right)-H(\sigma)\right]_{+}} & \text {if } \tau=\sigma^{(i)} \\ 1-\sum_{i} \frac{1}{L} e^{-\left[H\left(\sigma^{(i)}\right)-H(\sigma)\right]_{+}} & \text {if } \tau=\sigma \\ 0 & \text { otherwise }\end{cases}
$$

where $[\cdot]_{+}$means the positive part.

For both dynamics the one-dimensional stationary measure $\pi^{G}(\sigma)$ is well known. We have

$$
\pi^{G}(\sigma)=\frac{e^{-2 J \ell(\sigma)}}{2\left(1+e^{-2 J}\right)^{L-1}}
$$

where $\ell(\sigma)$ is the number of pair $\{i, i+1\}$ such that $\sigma_{i} \sigma_{i+1}=-1$ (i.e. $\ell(\sigma)$ is the total length of the Peierls contours).

We conclude this short discussion of the empty boundary conditions by checking the irreversibility of this dynamics, i.e., the presence of non zero probability currents. Indeed, for example, for $i>1$ and $m>1$ such that $i+m<L$, let us consider the configuration $\sigma$ with $\sigma_{j}=-1$ for $j=i, i+1, \ldots, i+m-1$ and $\sigma_{j}=+1$ elsewhere and observe that $\pi^{G}(\sigma)=\pi^{G}\left(\sigma^{(i)}\right)$ while $P\left(\sigma, \sigma^{(i)}\right)=\frac{1}{L}$ and $P\left(\sigma^{(i)}, \sigma\right)=\frac{e^{-4 J}}{L}$. Therefore

$$
\pi^{G}(\sigma) P\left(\sigma, \sigma^{(i)}\right)-\pi^{G}\left(\sigma^{(i)}\right) P\left(\sigma^{(i)}, \sigma\right)=\frac{1-e^{-4 J}}{L} \pi^{G}(\sigma)>0
$$

In order to control the invariant measure in the case of plus boundary conditions, we introduce a particular regime, defined as follows.

\section{Definition.}

We say that the one-dimensional discrete spin chain on $[1, L]$ with states $\sigma \in\{-1,+1\}\{1, \ldots, L\}$ subjected to the irreversible dynamics (3) or to the Glauber dynamics (10) is in the chilled regime of parameter $c>0$ if

$$
J=c \log L
$$

Note that the Gibbs measure $\pi^{G}$ for $c$ large enough is concentrated on the configurations $\sigma=\boxplus\left(\sigma_{i}=1 \forall i\right)$ and $\sigma=\boxminus\left(\sigma_{i}=-1 \forall i\right)$, while for the other configurations $\sigma$ we get

$$
\pi^{G}(\sigma) \sim \frac{1}{2} e^{-2 J \ell(\sigma)}
$$


The chilled condition defined above mimics a phase transition, in the sense that the volume dependent low temperature (high $J$ ) defined by $e^{-2 J} L \ll 1$ forces the system in a non zero (in particular, very close to \pm 1 ) magnetization. It is very easy, yet quite interesting, to study the mixing time of the two dynamics defined above, which is proportional to the expected value of the tunneling time, namely, the time needed to pass from the configuration $\boxplus$ to the configuration $\boxminus$.

It is not difficult to identify in the reversible case the typical path of the tunneling. By chilled condition $e^{-2 J} L \ll 1$, a spin flip on the boundary occurs after a time of the order of $L e^{2 J}$ and a spin flip inside a region of spins having all the same sign occurs after a time of the order of $e^{4 J}$. Both times are much longer than $L$. On the other side the interface between two regions with opposite spins may move in a time of order $L$, with equal probability on the right and on the left. Hence the typical path of the tunneling is a spin flip on one of the two boundaries followed by a random walk of the boundary between the + and the - regions which eventually reaches the other boundary. The latter event happens with probability $1 / L^{2}$, giving in the end a tunneling time of the order of $L^{3} e^{2 J}$. In the irreversible dynamics the spin in the site 1 is flipped after a time of the order $L e^{2 J}$. The boundary between the + and the - regions, then, typically moves only on the right, and this ensures that the tunneling time is of the order of $L e^{2 J}$, and hence shorter, polynomially in $L$, than the reversible case.

In what follows we will consider the case of + boundary conditions, namely $\sigma_{0}=\sigma_{L+1}=1$. With the reversible Glauber dynamics (10) the invariant measure with plus boundary conditions is just Gibbs measure $\pi^{G}$ conditioned to $\sigma_{0}=\sigma_{L+1}=1$. If we consider now the irreversible dynamics (3) we will see ahead that its invariant measure changes dramatically with respect to the free boundary conditions case.

For notational simplicity in the computation we will also use the notation $P_{\sigma \tau} \equiv P(\sigma, \tau)$ and $\pi_{\sigma} \equiv \pi(\sigma)$.

\subsection{Results}

Before stating our results concerning this particular regime we need to introduce the main technical tool which consists in writing the invariant measure of the irreversible dynamics

with + boundary conditions in the chilled regime in terms of a series in $e^{-4 J}$. We will omit for simplicity hereafter the suffix $I$, standing for irreversibility. 
Denoting with $\ell(\sigma)$ the number of antiparallel pairs of spins for each configuration $\sigma$ and recalling that $\sigma_{0}=1$, we can write the transition probability matrix in the following form

$$
P(\sigma, \tau)= \begin{cases}\frac{1}{L} & \text { if } \tau=\sigma^{(i)} \text { and } \sigma_{i} \sigma_{i-1}=-1 \\ \frac{e^{-4 J}}{L} & \text { if } \tau=\sigma^{(i)} \text { and } \sigma_{i} \sigma_{i-1}=1 \\ 1-\frac{\ell(\sigma)}{L}-\left(1-\frac{\ell(\sigma)}{L}\right) e^{-4 J} & \text { if } \tau=\sigma \\ 0 & \text { otherwise }\end{cases}
$$

We can define the dynamics above for zero temperature $(J \rightarrow \infty)$

$$
P^{(0)}(\sigma, \tau)= \begin{cases}\frac{1}{L} & \text { if } \tau=\sigma^{(i)} \text { and } \sigma_{i} \sigma_{i-1}=-1 \\ 1-\frac{\ell(\sigma)}{L} & \text { if } \tau=\sigma \\ 0 & \text { otherwise }\end{cases}
$$

obtaining

$$
P(\sigma, \tau)=P^{(0)}(\sigma, \tau)+e^{-4 J} \Delta P(\sigma, \tau)
$$

where

$$
\Delta P(\sigma, \tau)= \begin{cases}\frac{1}{L} & \text { if } \tau=\sigma^{(i)} \text { and } \sigma_{i} \sigma_{i-1}=1 \\ -1+\frac{\ell(\sigma)}{L} & \text { if } \tau=\sigma \\ 0 & \text { otherwise }\end{cases}
$$

The state $\sigma$ corresponding to $\ell(\sigma)=0$, i.e., $\sigma=\boxplus\left(\sigma_{i}=+1 \forall i\right)$, is clearly absorbent for the zero temperature dynamics. Hence

$$
\pi^{(0)}(\sigma)= \begin{cases}1 & \text { if } \sigma=\boxplus \\ 0 & \text { otherwise }\end{cases}
$$

We can use now the following formula for the perturbations on Markov chains:

$$
\pi(\sigma)=\sum_{k=0}^{\infty} e^{-4 J k} \pi^{(k)}(\sigma)
$$

where

$$
\pi^{(k)}(\sigma)=\sum_{\tau} \pi^{(0)}(\tau) D^{k}(\tau, \sigma) \quad D=\sum_{j=0}^{\infty} \Delta P\left(P^{(0)}\right)^{j}
$$

Again for notational simplicity we will write $P^{(0) j} \equiv\left(P^{(0)}\right)^{j}$. Note that by its definition

$$
\pi^{(k)}(\sigma)=0 \quad \forall \sigma: \ell(\sigma)>2 k
$$

Formulas (14) and (15) may be easily proved in general. Indeed, let $\pi_{i}^{(0)}$ the stationary 
measure of an ergodic Markov chain $P_{i j}^{(0)}$. Consider the chain $P_{i j}=P_{i j}^{(0)}+\varepsilon \Delta P_{i j}$. Denote with $\pi_{i}$ the stationary measure of the chain $P_{i j}$. By ergodic theorem we have

$$
\pi_{i}=\lim _{N \rightarrow \infty} \sum_{j} \pi_{j}^{(0)}\left(P_{i j}\right)^{N}=\lim _{N \rightarrow \infty} \sum_{j} \pi_{j}^{(0)}\left(P_{i j}^{(0)}+\varepsilon \Delta P_{i j}\right)^{N}
$$

Then defining

$$
D_{i j}=\sum_{l \geq 0} \sum_{k} \Delta P_{i k}\left(P^{(0) l}\right)_{k j}
$$

we have that

$$
\pi_{i}=\sum_{k} \pi_{i}^{(k)} \varepsilon^{k}
$$

with

$$
\pi_{i}^{(k)}=\sum_{l} \pi_{l}^{(0)}\left(D^{k}\right)_{l i}
$$

A similar expansion is used for instance in [3] for the blockage problem.

We define the expansion of the stationary measure up to the first order as

$$
\pi^{(\leq 1)}=\pi^{(0)}+e^{-4 J} \pi^{(1)}
$$

Note that $\pi^{(\leq 1)}$ is a probability measure.

We can now state our main results. The first is an immediate consequence of the convergence of the perturbative expansion (14). Let

$$
d_{T V}\left(\pi, \pi^{(\leq 1)}\right)=\sum_{\sigma}\left|\pi(\sigma)-\pi^{(\leq 1)}(\sigma)\right|
$$

be the total variation distance between the measure $\pi$ and its first order approximation $\pi^{(\leq 1)}$. Then the following theorem holds.

Theorem 2.1 In chilled regime of parameter $c=\frac{1}{2}+\gamma$, with $\gamma>0$, we have that

$$
d_{T V}\left(\pi, \pi^{(\leq 1)}\right) \leq \frac{\text { const }}{L^{8 \gamma}}
$$

Theorem 2.1 shows that it is meaningful, in the chilled regime with $\gamma>1 / 2$, to compute the first order in $e^{-4 J}$ of the stationary measure, since it will be the leading one. 
As it is clear from the perturbative approach, by (16), up to first order the only configurations admitted are the ones with at most one connected interval of sites having $\sigma_{i}=-1$, while all the rest of the configuration has $\sigma_{i}=+1$.

Let $i \in 1, \ldots, L-1$ and $m \in 1, \ldots, L-i$ and let us denote $(i ; m)$ the state having

$$
\sigma_{k}= \begin{cases}+1 & \text { for } 1 \leq k<i \\ -1 & \text { for } i \leq k<i+m \\ +1 & \text { for } i+m \leq k \leq L\end{cases}
$$

In other words $\sigma_{i} \sigma_{i-1}=-1, \sigma_{i+m} \sigma_{i+m-1}=-1, \sigma_{k} \sigma_{k-1}=1 \forall k \neq i, i+m$. That is, the state $(i ; m)$ is a single interval of $m$ spins equal to -1 starting at $i$,

Let us denote $(i)$ the state having

$$
\sigma_{k}= \begin{cases}+1 & \text { for } 1 \leq k<i \\ -1 & \text { for } i \leq k \leq L\end{cases}
$$

In other words $(i)=(i ; L+1-i)$, i.e., $\sigma_{i} \sigma_{i-1}=-1, \sigma_{k} \sigma_{k-1}=1 \forall k \neq i$.

Theorem 2.2 For any fixed $m>0$ and $i$ large we have

$$
\pi_{(i ; m)}^{(\leq 1)}=e^{-4 J}\left(1-\frac{C_{m}}{\sqrt{i}}+o\left(\frac{1}{\sqrt{i}}\right)\right)
$$

where $C_{m}$ is a constant depending on $m$. For every $i, m$ we have

$$
\pi_{(i ; m)}^{(\leq 1)} \leq 4 e^{-4 J} e^{-\frac{(m)^{2}}{2(i+m)}} m
$$

Moreover for every $i$

$$
\pi_{(i)}^{(\leq 1)}=\sum_{l=1}^{i} \pi_{(l ; L-l)}^{(\leq 1)}
$$

Remark. Note that by (19) we get $\pi_{(i ; m)}^{(\leq 1)} \rightarrow e^{-4 J}$ as $i \rightarrow \infty$, so that very far from the boundary condition the stationary distribution at the first order in $e^{-4 J}$ is equal to the Gibbs one, giving the same weight to every interval of minus spins independently of its length and its position. This convergence to the Gibbs measure, however, it is very slow, and it does not occur on a well defined scale. Moreover the exponential decay with the length $m$ of the interval of - spins given by (20) produces macroscopic effects, as the following theorem shows. 
Theorem 2.3 The average value of $m(\sigma):=\sum_{i=1}^{L} \mathbb{1}_{\left\{\left\{\sigma_{i}=-1\right\}\right\}}$ with respect to the Gibbs measure, $\pi^{G}$, and with respect to the irreversible measure up to the first order, $\pi^{(\leq 1)}$, are such that

$$
\lim _{L \rightarrow \infty} \frac{\pi^{(\leq 1)}(m)}{\pi^{G}(m)} \leq \frac{1}{4}
$$

\section{Proof of Theorem 2.1}

By (15) we have

$$
d_{T V}\left(\pi, \pi^{(\leq 1)}\right)=\sum_{\sigma}\left|\sum_{k=2}^{\infty} e^{-4 J k} \pi_{\sigma}^{(k)}\right| \leq \sum_{k=2}^{\infty} e^{-4 J k} \sum_{\sigma}\left|\pi_{\sigma}^{(k)}\right|
$$

For $J=c \log L$ the condition $c=\frac{1}{2}+\gamma$ implies $e^{-4 J k}=L^{-(2+4 \gamma) k}$ and then it is enough to prove that

$$
\sum_{\sigma}\left|\pi_{\sigma}^{(k)}\right| \leq\left(C L^{2}\right)^{k}
$$

Since

$$
\sum_{\sigma}\left|\pi_{\sigma}^{(k)}\right|=\sum_{\sigma}\left|\sum_{m=0}^{\infty} \sum_{\tau, \sigma^{\prime}} \pi_{\tau}^{(k-1)} \Delta P_{\tau \sigma^{\prime}}\left(P^{(0) m}\right)_{\sigma^{\prime} \sigma}\right|
$$

we have that (23) is recursively proved if we are able to prove that

$$
\sup _{\tau} \sum_{\sigma}\left|\sum_{m=0}^{\infty} \sum_{\sigma^{\prime}} \Delta P_{\tau \sigma^{\prime}}\left(P^{(0) m}\right)_{\sigma^{\prime} \sigma}\right| \leq C L^{2}
$$

Note first that

$$
\sum_{\sigma^{\prime}} \Delta P_{\tau \sigma^{\prime}}=0
$$

for all $\tau$. Then define the matrix $\Pi^{(0)}$, having all the rows equal to the stationary measure $\pi^{(0)}$, and hence having on the column related to the configuration $\sigma=\boxplus$, say on the first column, all the entries equal to 1, while all the other entries are zero. Observe that, due to (25), we have

$$
\sum_{\sigma^{\prime}} \Delta P_{\tau \sigma^{\prime}} \Pi_{\sigma^{\prime}, \tau}^{(0)}=0
$$

for all $\sigma$ and $\tau$. Finally define

$$
R_{m}=P^{(0) m}-\Pi^{(0)}
$$


Due to (26) we have that

$$
\sum_{\sigma^{\prime}} \Delta P_{\tau \sigma^{\prime}}\left(P^{(0) m}\right)_{\sigma^{\prime} \sigma}=\sum_{\sigma^{\prime}} \Delta P_{\tau \sigma^{\prime}}\left(R_{m}\right)_{\sigma^{\prime} \sigma}
$$

Now using (28) we split the sum over $m$ in two:

$$
\begin{gathered}
\sum_{\sigma}\left|\sum_{m=0}^{\infty} \sum_{\sigma^{\prime}} \Delta P_{\tau \sigma^{\prime}}\left(P^{(0) m}\right)_{\sigma^{\prime} \sigma}\right| \leq \\
\leq \sum_{\sigma}\left|\sum_{\sigma^{\prime}} \Delta P_{\tau \sigma^{\prime}}\left(\sum_{m=0}^{2 L^{2}} P^{(0) m}\right)_{\sigma^{\prime} \sigma}\right|+\sum_{\sigma}\left|\sum_{\sigma^{\prime}} \Delta P_{\tau \sigma^{\prime}}\left(\sum_{m=2 L^{2}+1}^{\infty} R_{m}\right)_{\sigma^{\prime} \sigma}\right|
\end{gathered}
$$

The first term is estimated as follows

$$
\begin{gathered}
\sum_{\sigma}\left|\sum_{\sigma^{\prime}} \Delta P_{\tau \sigma^{\prime}}\left(\sum_{m=0}^{2 L^{2}} P^{(0) m}\right)_{\sigma^{\prime} \sigma}\right| \leq \sum_{\sigma, \sigma^{\prime}}\left|\Delta P_{\tau \sigma^{\prime}}\left(\sum_{m=0}^{2 L^{2}} P^{(0) m}\right)_{\sigma^{\prime} \sigma}\right| \leq \\
\leq \sum_{\sigma^{\prime}}\left|\Delta P_{\tau \sigma^{\prime}}\right| \sum_{m=0}^{2 L^{2}} \sum_{\sigma}\left(P^{(0) m}\right)_{\sigma^{\prime} \sigma}
\end{gathered}
$$

The sum on $\sigma$ is 1 for each addend of the sum on $m$, and then

$$
\sum_{\sigma}\left|\sum_{\sigma^{\prime}} \Delta P_{\tau \sigma^{\prime}}\left(\sum_{m=0}^{2 L^{2}} P^{(0) m}\right)_{\sigma^{\prime} \sigma}\right| \leq \sum_{\sigma^{\prime}}\left|\Delta P_{\tau \sigma^{\prime}}\right| 2 L^{2}
$$

Since, due to the definition of $\Delta P_{\tau \sigma^{\prime}}$, we have

$$
\sum_{\sigma^{\prime}}\left|\Delta P_{\tau \sigma^{\prime}}\right|=2\left(1-\frac{\ell(\tau)}{L}\right) \leq 2
$$

we obtain the following estimate

$$
\sum_{\sigma}\left|\sum_{\sigma^{\prime}} \Delta P_{\tau \sigma^{\prime}}\left(\sum_{m=0}^{2 L^{2}} P^{(0) m}\right)_{\sigma^{\prime} \sigma}\right| \leq 4 L^{2}
$$

Now we are left with the estimate of the second term in (29):

$$
\sum_{\sigma}\left|\sum_{\sigma^{\prime}} \Delta P_{\tau \sigma^{\prime}}\left(\sum_{m=2 L^{2}+1}^{\infty} R_{m}\right)_{\sigma^{\prime} \sigma}\right| \leq \sum_{\sigma, \sigma^{\prime}}\left|\Delta P_{\tau \sigma^{\prime}}\left(\sum_{m=2 L^{2}+1}^{\infty} R_{m}\right)_{\sigma^{\prime} \sigma}\right|
$$


Let us first of all consider the entries of the matrix $R_{m}$. Calling $T_{\boxplus}\left(\sigma^{\prime}\right)$ the hitting time to the state $\boxplus$ starting from the state $\sigma^{\prime}$ we have that, being $\boxplus$ an absorbent state,

$$
\left(R_{m}\right)_{\sigma^{\prime}, \boxplus}=P_{\sigma^{\prime}, \boxplus}^{(0) m}-1=-P\left(T_{\boxplus}\left(\sigma^{\prime}\right)>m\right)
$$

For the same reason

$$
\sum_{\sigma \neq \boxplus}\left(R_{m}\right)_{\sigma^{\prime}, \sigma}=P\left(T_{\boxplus}\left(\sigma^{\prime}\right)>m\right)
$$

and therefore

$$
\sum_{\sigma}\left|\left(R_{m}\right)_{\sigma^{\prime}, \sigma}\right|=2 P\left(T_{\boxplus}\left(\sigma^{\prime}\right)>m\right)
$$

Hence

$$
\begin{aligned}
& \sum_{\sigma, \sigma^{\prime}}\left|\Delta P_{\tau \sigma^{\prime}}\left(\sum_{m=2 L^{2}+1}^{\infty} R_{m}\right)_{\sigma^{\prime} \sigma}\right| \leq 2 \sum_{\sigma^{\prime}}\left|\Delta P_{\tau \sigma^{\prime}} \sum_{m=2 L^{2}+1}^{\infty} P\left(T_{\boxplus}\left(\sigma^{\prime}\right)>m\right)\right| \leq \\
\leq & 2\left(\sup _{\sigma^{\prime}} \sum_{m=2 L^{2}+1}^{\infty} P\left(T_{\boxplus}\left(\sigma^{\prime}\right)>m\right)\right) \sum_{\sigma^{\prime}}\left|\Delta P_{\tau \sigma^{\prime}}\right| \leq \underset{\sigma^{\prime}}{4} \sup _{m=2 L^{2}+1}^{\infty} P\left(T_{\boxplus}\left(\sigma^{\prime}\right)>m\right)
\end{aligned}
$$

where in the last line we used again (30).

We are left with an estimate uniform in $\sigma^{\prime}$ of the quantity $P\left(T_{\boxplus}\left(\sigma^{\prime}\right)>m\right)$. Recall that the (zero temperature) dynamics chooses u.a.r. a site and try to update it. Call $\xi_{1}$ the time needed to choose for the first time the site 1 , then $\xi_{2}$ the time needed, after the first choose of the site 1 , to choose for the first time the site 2, and so on so forth. Calling $\xi=\sum_{i=1}^{L} \xi_{i}$ we have that $\xi \geq T_{\boxplus}\left(\sigma^{\prime}\right)$ for all $\sigma^{\prime}$. This is granted by the fact that after the time $\xi_{1}$ we have definitively that $\sigma_{1}=+1$, after the time $\xi_{1}+\xi_{2}$ we have definitively that $\sigma_{1}=\sigma_{2}=+1$ and so on. Hence we have for all $\sigma^{\prime}$

$$
P\left(T_{\boxplus}\left(\sigma^{\prime}\right)>m\right) \leq P(\xi>m)
$$

Being $\xi_{i}$ a geometrical variable of probability $p=\frac{1}{L}$, and hence having $E\left(\xi_{i}\right)=L$, $\operatorname{Var}\left(\xi_{i}\right)=L^{2}$ for all $i$, we have that $\xi$ is the sum of $L$ independent geometric identical variables, and therefore $E(\xi)=L^{2}, \operatorname{Var}(\xi)=L^{3}$.

By Chebyshev inequality

$$
P(\xi>m)=P(\xi-E(\xi)>m-E(\xi))=P\left(\xi-E(\xi)>m-L^{2}\right) \leq \frac{L^{3}}{\left(m-L^{2}\right)^{2}}
$$


We have then proved that

$$
\sup _{\sigma^{\prime}} \sum_{m=2 L^{2}+1}^{\infty} P\left(T_{\boxplus}\left(\sigma^{\prime}\right)>m\right) \leq \sum_{m=2 L^{2}+1}^{\infty} \frac{L^{3}}{\left(m-L^{2}\right)^{2}} \leq L
$$

which finally gives

$$
\sum_{\sigma}\left|\sum_{\sigma^{\prime}} \Delta P_{\tau \sigma^{\prime}}\left(\sum_{m=2 L^{2}+1}^{\infty} R_{m}\right)_{\sigma^{\prime} \sigma}\right| \leq 4 L
$$

Combining (32) and (31) we get (24).

\section{Proof of Theorems 2.2 and 2.3}

Let us denote with $\lambda((k ; 1),(i ; m))$ a sequence of spin flip, allowed by the zero temperature dynamics, that brings the configuration $(k ; 1)$ into the configuration $(i ; m)$. Since at least one - spin has to be present in all the steps of the sequence, the latter can be described by partial Dyck words, and the number of such sequence is given by the elements of the so-called Catalan's triangle (see e.g. [1], [13]).

We have

$$
\begin{gathered}
\pi_{(i ; m)}^{(1)}=D_{+,(i ; m)}=\frac{1}{L} \sum_{k=1}^{i} \sum_{s=0}^{\infty} P_{(k ; 1),(i ; m)}^{(0) s}=\frac{1}{L} \sum_{k=1}^{i} \sum_{s=2 i+m-2 k-1}^{\infty} P_{(k ; 1),(i ; m)}^{(0) s}= \\
=\frac{1}{L} \sum_{k=1}^{i} \frac{1}{L^{2 i+m-2 k-1}} \sum_{\lambda((k ; 1),(i ; m))} \sum_{s^{\prime}=0}^{\infty}\left(\begin{array}{c}
2 i+m-2 k-1+s^{\prime} \\
s^{\prime}
\end{array}\right)\left(1-\frac{2}{L}\right)^{s^{\prime}}= \\
=\sum_{k=1}^{i} \frac{1}{L^{2 i+m-2 k}}\left(\frac{L}{2}\right)^{2 i+m-2 k} C_{i+m-k-1, i-k}=\sum_{k=1}^{i}\left(\frac{1}{2}\right)^{2 i+m-2 k} C_{i+m-k-1, i-k}
\end{gathered}
$$

where in the second line we defined $s^{\prime}=s-2 i-m+2 k+1$, and in the last line we used the Taylor expansion, convergent for $|\alpha|<1$, of the function $\left(\frac{1}{1-\alpha}\right)^{N+1}$

$$
\left(\frac{1}{1-\alpha}\right)^{N+1}=\sum_{s=0}^{\infty}\left(\begin{array}{c}
N+s \\
s
\end{array}\right) \alpha^{s}
$$

In equation (33) $C_{i+m-k-1, i-k}$ denotes the number appearing in the position $i+m-k-$ $1, i-k$ of the Catalan's triangle, i.e.

$$
C_{n, k}=\frac{(n+k) !(n-k+1)}{k !(n+1) !} .
$$


Calling $l=i-k$ we have

$$
\pi_{(i ; m)}^{(1)}=\sum_{l=0}^{i-1}\left(\frac{1}{2}\right)^{2 l+m} C_{l+m-1, l}
$$

We will now prove the following lemma.

Lemma 4.1 For every positive integer $m$ we have

$$
\sum_{l=0}^{\infty}\left(\frac{1}{2}\right)^{2 l+m} C_{l+m-1, l}=1
$$

Proof. The quantity $\pi_{(i ; m)}^{(1)}$ can be written in terms of a one dimensional Symmetric Random Walk (SRW), $S_{n}=\sum_{i=1}^{n} X_{i}$, with $X_{i}$ independent Bernoulli variables $X_{i} \in$ $\{-1,+1\}$. Indeed $C_{l+m-1, l}$ is the number of paths of the random walk $\left\{S_{n}\right\}_{n \in \mathbb{N}}$ such that $S_{1}=1, S_{2 l+m}=m$ and $S_{n}>0$ for any $n=1, \ldots, 2 l+m$. For the duality principle for random walks, we have that $\left(X_{1}, X_{2}, \ldots, X_{n}\right)$ has the same distribution of $\left(X_{n}, X_{n-1}, \ldots, X_{1}\right)$, so that the path $\left(0, S_{1}, S_{2}, \ldots, S_{n}\right)$ has the same probability of the time reversal path $\left(0, S_{n}-S_{n-1}, S_{n}-S_{n-2}, \ldots, S_{n}-0\right)$. This implies that by denoting with $\tau_{m}$ the first hitting time to $m$ for the random walk starting at 0 , we have for every positive integer $m$

$$
\left(\frac{1}{2}\right)^{2 l+m} C_{l+m-1, l}=P\left(\tau_{m}=2 l+m\right)
$$

so that

$$
\pi_{(i ; m)}^{(1)}=\sum_{l=0}^{i-1} P\left(\tau_{m}=2 l+m\right)=P\left(\tau_{m}<2 i+m\right) .
$$

Formula (36) now immediately follows from (38) since for the SRW the hitting of any state is finite with probability one.

Remark. The proof of (36) can also be obtained in a purely combinatorial framework. See for instance Lemma 18 in reference [10].

We now prove (19). From (35) and Lemma 4.1 we have

$$
\pi_{(i ; m)}^{(1)}=1-\sum_{l=i}^{\infty}\left(\frac{1}{2}\right)^{2 l+m} C_{l+m-1, l}
$$

with

$$
\left(\frac{1}{2}\right)^{2 l+m} C_{l+m-1, l}=\left(\frac{1}{2}\right)^{2 l+m} \frac{(2 l+m) !}{(l+m) ! l !} \frac{m}{2 l+m}
$$


Using upper and lower Stirling's bounds for the factorials [12] valid for all $n \geq 1$

$$
\sqrt{2 \pi n}\left(\frac{n}{e}\right)^{n} e^{\frac{1}{12 n+1}}<n !<\sqrt{2 \pi n}\left(\frac{n}{e}\right)^{n} e^{\frac{1}{12 n}}
$$

we have, for any $l \geq 1$ and any $m \geq 1$

$$
\begin{gathered}
\left(\frac{1}{2}\right)^{2 l+m} C_{l+m-1, l} \leq \frac{e^{\frac{1}{12}}}{\sqrt{2 \pi}} \frac{\left(1+\frac{m}{2 l}\right)^{2 l+m}}{\left(1+\frac{m}{l}\right)^{l+m}} \frac{m}{\sqrt{l(l+m)(2 l+m)}} \leq \\
\leq \frac{e^{\frac{1}{12}}}{\sqrt{2 \pi}}\left(\frac{1+\frac{m}{2 l}}{1+\frac{m}{l}}\right)^{m}\left(\frac{\left(1+\frac{m}{2 l}\right)^{2}}{1+\frac{m}{l}}\right)^{l} \frac{m}{\sqrt{l(l+m)(2 l+m)}} \leq \\
\leq \frac{e^{\frac{1}{12}}}{\sqrt{2 \pi}}\left(\frac{l+\frac{m}{2}}{l+m}\right)^{m}\left(1+\frac{m}{l}\right) \frac{m}{\sqrt{l(l+m)(2 l+m)}}= \\
=\frac{e^{\frac{1}{12}}}{\sqrt{2 \pi}}\left(1-\frac{m}{2(l+m)}\right)^{m} \frac{m}{l^{3 / 2}} \sqrt{\frac{l+m}{2 l+m}} \\
\leq \frac{1}{2} e^{\frac{-m^{2}}{2(m+l)}} \frac{m}{l^{3 / 2}}
\end{gathered}
$$

where in the last line we have used the trivial bound $(1-x) \leq e^{-x}$ valid for all $x \geq 0$. Hence for any $l \geq 1$ and any $m \geq 1$ we may roughly bound

$$
\left(\frac{1}{2}\right)^{2 l+m} C_{l+m-1, l} \leq \frac{m}{2} \frac{1}{l^{3 / 2}}
$$

A similar computation gives, for any $l \geq 1$ and any $m \geq 1$,

$$
\begin{gathered}
\left(\frac{1}{2}\right)^{2 l+m} C_{l+m-1, l} \geq \frac{e^{-\frac{1}{6}}}{\sqrt{2 \pi}} \frac{\left(1+\frac{m}{2 l}\right)^{2 l+m}}{\left(1+\frac{m}{l}\right)^{l+m}} \frac{m}{\sqrt{l(l+m)(2 l+m)}} \geq \\
\geq \frac{1}{3}\left(\frac{1+\frac{m}{2 l}}{1+\frac{m}{l}}\right)^{m}\left(\frac{\left(1+\frac{m}{2 l}\right)^{2}}{1+\frac{m}{l}}\right)^{l} \frac{m}{\sqrt{l(l+m)(2 l+m)}} \geq \\
\geq \frac{1}{3}\left(\frac{l+\frac{m}{2}}{l+m}\right)^{m} \frac{m}{\sqrt{l(l+m)(2 l+m)}}=
\end{gathered}
$$

Therefore we may roughly bound for any $l \geq 1$ and any $m \geq 1$

$$
\left(\frac{1}{2}\right)^{2 l+m} C_{l+m-1, l} \geq \frac{2^{-m}}{3 \sqrt{6}} \frac{1}{l^{3 / 2}}
$$


From inequalities (40) and (41) the first statement (19) of Theorem 2.2 immediately follows.

In order to show (20) we write

$$
\pi_{(i ; m)}^{(1)}=\left(\frac{1}{2}\right)^{m}+\sum_{l=1}^{i-1}\left(\frac{1}{2}\right)^{2 l+m} \frac{(2 l+m) !}{(l+m) ! l !} \frac{m}{2 l+m}
$$

Using now (40) and recalling that $\sum_{n=1}^{\infty} \frac{1}{n^{3 / 2}}=\zeta(3 / 2) \leq 3$, we get

$$
\begin{aligned}
\pi_{(i ; m)}^{(1)} & \leq\left(\frac{1}{2}\right)^{m}+\frac{1}{2} \sum_{l=1}^{i-1} e^{\frac{-m^{2}}{2(m+l)}} \frac{m}{l^{3 / 2}} \leq\left(\frac{1}{2}\right)^{m}+\frac{e^{\frac{-m^{2}}{2(m+i)}}}{2} \sum_{l=1}^{\infty} \frac{m}{l^{3 / 2}} \leq \\
& \leq\left(\frac{1}{2}\right)^{m}+3 m e^{\frac{-m^{2}}{2(m+i)}} \leq(1+3 m) e^{\frac{-m^{2}}{2(m+i)}} \leq 4 m e^{\frac{-m^{2}}{2(m+i)}}
\end{aligned}
$$

and inserting (42) inequality into (39) we get (20).

The computation of $\pi_{(i)}^{(1)}$ is similar, but it is necessary to choose the time in which the spin in the site $L$ is flipped to $\sigma_{L}=-1$. We have

$$
\begin{gathered}
\pi_{(i)}^{(1)}=D_{+,(i)}=\frac{1}{L} \sum_{k=1}^{i} \sum_{m=0}^{\infty} P_{(k ; 1),(i)}^{(0) m}= \\
=\frac{1}{L} \sum_{k=1}^{i} \sum_{l=k}^{i} \frac{1}{L^{L+l-2 k-1}} \sum_{\lambda((k ; 1),(l ; L-l))} \sum_{m^{\prime}=0}^{\infty}\left(\begin{array}{c}
L+l-2 k-1+m^{\prime} \\
m^{\prime}
\end{array}\right)\left(1-\frac{2}{L}\right)^{m^{\prime}} \times \\
\times \frac{1}{L} \frac{1}{L^{i-l}} \sum_{m^{\prime \prime}=0}^{\infty}\left(\begin{array}{c}
i-l+m^{\prime \prime} \\
m^{\prime \prime}
\end{array}\right)\left(1-\frac{i}{L}\right)^{m^{\prime \prime}}=\sum_{k=1}^{i} \sum_{l=k}^{i}\left(\frac{1}{2}\right)^{L+l-2 k} C_{L-k-1, l-k}= \\
=\sum_{l=1}^{i} \sum_{k=1}^{l}\left(\frac{1}{2}\right)^{L+l-2 k} C_{L-k-1, l-k}=\sum_{l=1}^{i} \pi_{(l ; L-l)}^{(1)}
\end{gathered}
$$

This ends the proof of Theorem 2.2 .

To prove Theorem 2.3 we first observe that in the chilled regime the Gibbs measure $\pi^{G}(\sigma)$ is such that

$$
\pi^{G}(\sigma)=\frac{e^{-2 J \ell(\sigma)}}{1+o(1)}
$$

where $o(1)$ denotes any function of $L$ such that $\lim _{L \rightarrow \infty} o(1)=0$. So if we let

$$
\widehat{\pi}^{G}(\sigma)=e^{-2 J \ell(\sigma)}
$$


we have clearly that

$$
\lim _{L \rightarrow \infty} \frac{\pi^{(\leq 1)}(m)}{\pi^{G}(m)}=\lim _{L \rightarrow \infty} \frac{\pi^{(\leq 1)}(m)}{\widehat{\pi}^{G}(m)}
$$

We start computing $\widehat{\pi}^{G}(m)$. Observe that

$$
\widehat{\pi}^{G}(m)=e^{-4 J} \sum_{i=1}^{L} \sum_{m=1}^{L-i} m+\sum_{m=1}^{L} m \sum_{k=2}^{L / 2} e^{-4 k J} n(k, m)
$$

where $n(k, m)$ is the number of configurations with $k$ disjoint intervals of minus spins with a total number $m$ of minus spins. Due to the rough estimate $n(k, m)<L^{2 k-1}$ we get

$$
\widehat{\pi}^{G}(m) \leq\left[\frac{e^{-4 J}}{6}\left(L^{3}-L\right)+L^{3} e^{-4 J} o(1)\right] \leq \frac{L^{3} e^{-4 J}}{6}(1+o(1))
$$

We next estimate the difference $\widehat{\pi}^{G}(m)-\pi^{(\leq 1)}(m)$. Observe that by (44)

$$
\widehat{\pi}^{G}(m) \geq e^{-4 J} \sum_{i=1}^{L} \sum_{m=1}^{L-i} m
$$

and that by (16) and (20)

$$
\pi^{(\leq 1)}(m)=\pi_{(i ; m)}^{(\leq 1)}=e^{-4 J} \pi_{(i ; m)}^{(1)}
$$

so we have

$$
\widehat{\pi}^{G}(m)-\pi^{(\leq 1)}(m) \geq e^{-4 J} \sum_{i=1}^{L} \sum_{m=1}^{L-i} m\left(1-\pi_{(i ; m)}^{(1)}\right)
$$

Then note that, due to (39) we have that $1-\pi_{(i ; m)}^{(1)}>0$, so we are allowed to restricted the sums over $i, m$ above to a subset in which $i \leq m$. Recalling also bound (20) we get

$$
\begin{aligned}
\widehat{\pi}^{G}(m)-\pi^{(\leq 1)}(m) & \geq e^{-4 J} \sum_{i=1}^{L / 2} \sum_{m=i}^{L-i} m\left(1-\pi_{(i ; m)}^{(1)}\right) \geq e^{-4 J} \sum_{i=1}^{L / 2} \sum_{m=i}^{L-i}\left(m-4 e^{-\frac{m^{2}}{2(i+m)}} m^{2}\right) \\
& \geq e^{-4 J} \sum_{i=1}^{L / 2} \sum_{m=i}^{L-i}\left(m-4 e^{-\frac{m}{4}} m^{2}\right) \geq \frac{e^{-4 J} L^{3}}{8}(1+o(1))
\end{aligned}
$$

Hence, from inequalities (45) and (46) we get

$$
\frac{\widehat{\pi}^{G}(m)-\pi^{(\leq 1)}(m)}{\widehat{\pi}^{G}(m)} \geq \frac{3}{4}(1+o(1))
$$


whence

$$
\lim _{L \rightarrow \infty} \frac{\pi^{(\leq 1)}(m)}{\widehat{\pi}^{G}(m)} \leq \frac{1}{4}
$$

and from (43) Theorem 2.3 immediately follows.

\section{Conclusions}

In this paper we have considered an example of a single spin flip irreversible dynamics for a system very simple, but yet quite difficult to study in presence of boundary conditions. With explicit estimates we have shown that, expanding in series the stationary measure around the zero temperature, it is possible to control for very low temperature the convergence of the expansion and to compute, up to the first order, the stationary probability distribution. The latter has non trivial features: it has an explicit dependence both on the relative distance and on the position of the changes of sign in the state. Moreover the memory of the boundary conditions has a very slow decay, and crucial macroscopic effects.

There are several questions opened by this result. The generalization of this computations to PCA dynamics, like the one presented in [4] and [5], should be straightforward. It should be possible also, with some extra effort, to understand the features of the higher terms of the expansion, and it would be very interesting to generalize this technique to higher dimensions. All these questions will be the subject of further investigations.

Acknowledgments: We thank Pietro Caputo for equation (37) and Francesco Pappalardi for useful discussions on the combinatorial properties of Catalan's triagles, and related references. We also thank A.De Sole, D.Gabrielli, G.Gallavotti, G.Jona-Lasinio for useful discussions. A.P. has been partially supported by Conselho Nacional de Desenvolvimento Científico e Tecnológico (CNPq), Fundação de Amparo à Pesquisa do Estado de Minas Gerais (FAPEMIG - Programa de Pesquisador Mineiro) and by the Simons Foundation and the Mathematisches Forschungsinstitut Oberwolfach. His stay in Rome during part of this work has been founded by the Grant "Visiting Professors" of the Universita' di Roma Tor Vergata. B.S. and E.S. thank the support of the A*MIDEX project (n. ANR-11-IDEX0001-02) funded by the "Investissements d'Avenir" French Government program, managed by the French National Research Agency (ANR). B.S. has been supported by PRIN 2012, Problemi matematici in teoria cinetica ed applicazioni. E.S. has been supported by the 
PRIN 20155PAWZB "Large Scale Random Structures".

\section{References}

[1] D. F. BAYley, Counting Arrangements of 1's and -1's, Mathematical Magazine, 69, 128,131 (1996).

[2] T. Chou, K. Mallick, And R. K. P. ZI, Non-Equilibrium Statistical Mechanics: From a Paradigmatic Model to Biological Transport, Rep. Prog. Phys., 74, 116601 (2011).

[3] O. Costin, J. L. Lebowitz, E. R. Speer, and A. Troiani, The blockage problem, Bull. Inst. Math. Acad. Sin. N. S., 8, 49-72 (2013).

[4] P.Dai Pra, B.Scoppola, E.Scoppola Sampling from a Gibbs measure with pair interaction by means of PCA, J. Statist. Phys., 149, 722-737 (2012).

[5] P.Dai Pra, B.Scoppola, E.Scoppola Fast mixing for the low-temperature 2D Ising model through irreversible parallel dynamics J. Statist. Phys., 159, 1-20 (2015).

[6] L.De Carlo, D.Gabrielli, Gibbsian stationary non equilibrium states, arXiv:1703.02418v1.

[7] G. Gallavotti, Nonequilibrium and irreversibility, Springer-Verlag, Heidelberg (2014)

[8] A.Gaudillière, C. Landim, A Dirichlet principle for non reversible Markov chains and some recurrence theorems, Probab. Theory Related Fields, 158, 55-89 (2013).

[9] A. Kolmogorov, Zur Theorie der Markoffschen Ketten, Math. Ann., 112, 155-160 (1936).

[10] S. A. NG, Some identities and formulas involving generalized Catalan numbers, arXiv:math/0609596w1 (2006).

[11] A. Procacci, B. Scoppola, E. Scoppola, Probabilistic Cellular Automata for the low-temperature 2d Ising Model, J. Statist. Phys., 165, 991-1005 (2016).

[12] H. Robbins, A Remark on Stirlings Formula, Amer. Math. Monthly, 62, 26-29 (1955). 
[13] E. W. Weisstein, Catalan's Triangle MathWorld - A Wolfram Web Resource. Retrieved March 28, (2012). 